\title{
One Dimensional Shock Refraction at a Plasma-cold Gas Interface
}

\author{
J. Gerofi and H. K. Messerle * \\ University of Sydney (Australia) \\ (Z. Naturforsch. 28 a, 1668-1675 [1973] ; received 22 March 1973)
}

\begin{abstract}
Refraction and reflection of a shockwave at a plasma/cold gas interface has been studied using an R.F. preheated section in an electrothermal shock tube. The gas used in the experiment was Argon at initial pressures from 10 to 30 Torr, with initial temperature of $9000 \mathrm{~K}$.

A detailed numerical analysis of the refraction event has been undertaken using a method that does not require definition of an effective $\gamma$. Methods that do use such a $\gamma$ are not accurate, except for very weak shocks. Because of short ionization times it has been possible to assume equilibrium behind the various shock waves. Calculations suggest that in the region of interest, reflected and refracted shock velocities depend primarily on initial shock velocity, slightly on initial plasma temperature and very weakly on initial pressure. The analysis covers initial temperatures of 6000 to $12000 \mathrm{~K}$ and initial pressures of 10 to 50 Torr.

Calculations and experimental results are presented. These show that a step function discontinuity is a good approximation to the nature of the plasma cold gas interface in this situation.
\end{abstract}

\section{Introduction}

Electrothermal shock tubes are those in which shock waves are developed by heating the gas at one end of the tube with a capaciter bank discharge and allowing the plasma so formed to expand into the test region ${ }^{1,2}$. Recently, electrothermal driven shocks have been made to propagate through an induction pre-heated plasma. The preheating of the downstream gas is achieved using a $40 \mathrm{~kW}$ Radio Frequency Oscillator. The advantages of this system are that shock speed attenuation is lower than in a cold test gas configuration and the shock structure can be much better developed. Temperatures achieved are also higher for similar incident shock speeds.

Previous analyses of shock refractions and similar events (as discussed in more detail below) have either dealt only with ideal gases ${ }^{3}$, or used ideal gas type analyses by defining an effective $\gamma$ for the gas under consideration ${ }^{4}$. Such methods are only suitable for weak shocks, whereas the method used here has general application.

The related problem of shock collision has been studied by Strachan and Ahlborn ${ }^{5}$, and Bowman and Whittaker ${ }^{6}$. Strachan and Ahlborn's method of analysis is suitable only for a strong incident shock, and involves a semi-graphical approach. Bowman and Whittaker have used colliding shocks to deter-

\footnotetext{
* Professor Messerle is currently visiting Professor at Stuttgart University.
}

mine arc temperatures, but their calculations depend on ionization and dissociation being frozen and on the shocks being weak.

In this paper real gas effects are taken into account and numerical and experimental results for the behaviour of shock waves at a plasma boundary in the electrothermal shock tube are compared.

\section{Experimental Conditions and Apparatus}

The R. F. shock tube with its water cooled heating coils is shown in Fig. $1 \mathrm{a}$, while Fig. $1 \mathrm{~b}$ gives the schematic diagram of the tube. The inner

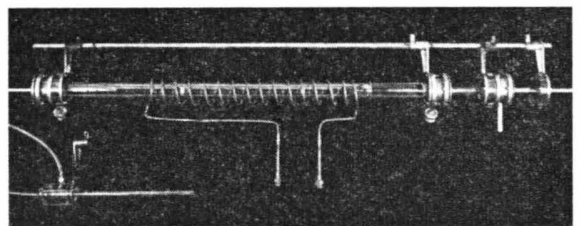

Fig. 1 a

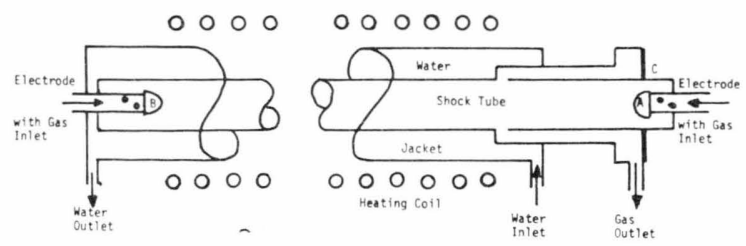

Fig. $1 \mathrm{~b}$

Fig. 1. a) Photograph of the RF Shock Tube with watercooled reflecting plane shown below. b) Schematic diagram of the RF Shock Tube.

Reprint requests to Prof. Dr. H. K. Messerle, Institut für Plasmaforschung der Universität Stuttgart, D-7000 Stuttgart 80 , Pfaffenwaldring 31. 
tube ob about $22 \mathrm{~mm}$. in diameter is made of silica (Vycor) and the overall length of the shock tube is about $100 \mathrm{~cm}$. It has three electrodes, A, B and C (Figure $1 \mathrm{~b}$ ). A and $\mathrm{B}$ are made of Carbon while $\mathrm{C}$ is a brass vacuum fitting. The central part of the shock tube is heated by a $40 \mathrm{~kW}$ Radio Frequency (R.F.) oscillator. Initially, at either end of the tube about $20 \mathrm{~cm}$ length of the gas remains almost at the room temperature, while the central part of the gas column is at a temperature of approximately $9000 \mathrm{~K}$. Gas-dynamic shocks are initiated by discharging a capacitor bank into specified regions in the tube. This may be done in two ways, by connecting the capacitor bank across either $\mathrm{AB}$ or $\mathrm{AC}$. The $\mathrm{AB}$ discharge results in dumping the capacitor energy into the whole of the tube. This simultaneously produces two shock waves at both the coldhot gas interfaces and the waves then travel towards the centre of the tube at which they interact (double ended mode). The discharge across AC initiates only a single shock from the cold-hot gas interface at that end and the wave travels through the entire tube (single ended mode). It is in this single ended mode that experiments described here are conducted.

The rapid arc heating in the right hand end of the tube, when the discharge is connected across $\mathrm{AC}$, produces a shock wave which travels down the tube towards B. If the right hand half of the R.F. coil is short-circuited, then the shock travels first through cold gas and then into the plasma. If the left hand half is short circuited, then the shock travels through the plasma into cold gas. This allows for an experimental study of the interaction of the shock with a fairly sharp discontinuity in density, provided by the hot-cold or cold-hot interface.

Observations are made by using a streak camera which gives $x-t$ diagrams showing the positions of luminous fronts in the shock tube. An Imacon image converter camera which is capable of producing a static picture of the tube section also has been used for the study.

Experiments have been carried out in argon at initial pressures of 30 Torr to 10 Torr and the photographic records were taken for a series of exposures at capacitor voltages of $2 \mathrm{kV}$ to $12 \mathrm{kV}$. The R.F. power has been kept constant at $7 \mathrm{~kW}$ throughout the experimental study. At 30 Torr the R.F. discharge produces a plasma with only very slightly enhanced electron temperature. Non-equilibrium effects in the unshocked plasma increase as pressure is reduced, but the pressure behind the shock is much higher than that in front.

Blackman and Niblett ${ }^{7}$, have measured ionization times behind shocks in argon of similar purity to ours. Their results, when applied to our situation suggest relaxation times of less than $20 \mu \mathrm{sec}$ in all cases considered and less than $10 \mu \mathrm{sec}$ when the incident shock mach number is greater than 1.7. However, because our initial conditions involve the argon being $1 \%$ ionized before the shock is fired, there will already be an appreciable downstream electron density behind the shock before equilibrium is reached. These electrons will make the ionization process considerably speedier than it was in Blackman and Niblett's case, as they fired shocks into argon at room temperature. In view of this it seems reasonable to assume equilibrium behind the shocks propagating into plasma.

\section{Refraction of an Incident Shock Wave}

When a shock wave is incident on a sharp discontinuity in gas properties (interface), it will be transmitted through the discontinuity and a reflected wave may develop at the interface. The reflected wave can be a compression wave in which case it sharpens into a shock wave, or a rarefaction wave. Two $x-t$ diagrams showing schematically a reflected shock wave and a reflected rarefaction wave, are given in Fig. 2 a and Figure 2 b. The initial conditions in the upstream region are denoted by subscript 0 and those in the downstream region by 3 . The conditions behind the incident shock are represented by 1 , those behind the refracted shock by 4 and those behind the reflected wave by 2 , (Figures $2 \mathrm{a}$ and $2 \mathrm{~b}$ ). $P, T$ and $\varrho$ are the gas pressure, temperature and density. $U$ is the shock front velocity and $u$ is the gas velocity behind the shock wave. $c$ is the speed of sound and $\gamma=c_{p} / c_{v}$ is the ratio of the specific heats of the gas at constant pressure $\left(c_{p}\right)$ and constant volume $\left(c_{v}\right)$.

The incident shock strength is defined by $y=$ $P_{1} / P_{0}$, the reflected shock strength $z=P_{2} / P_{1}$ and the transmitted shock strength $x=P_{4} / P_{3}$. For establishing a reflected wave, two boundary conditions are assumed,

$$
\text { (i) } P_{4}=P_{2} \text {. }
$$

Because $P_{3}=P_{0}$ in our experiments we can write $x=y z$.

$$
\text { (ii) } u_{4}=u_{2} \text {. }
$$

It should be noted that the two boundary conditions cannot be satisfied simultaneously if $z=1$ (which would mean that there is no reflected wave). For refraction between two ideal gases of equal $\gamma$, we 


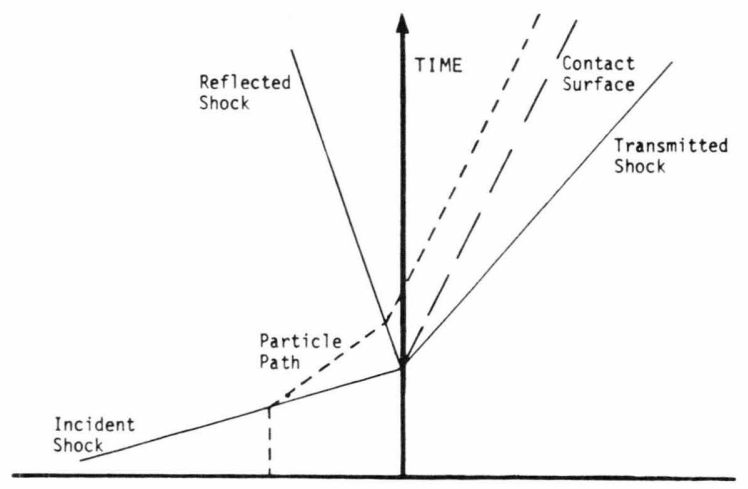

Fig. 2 a

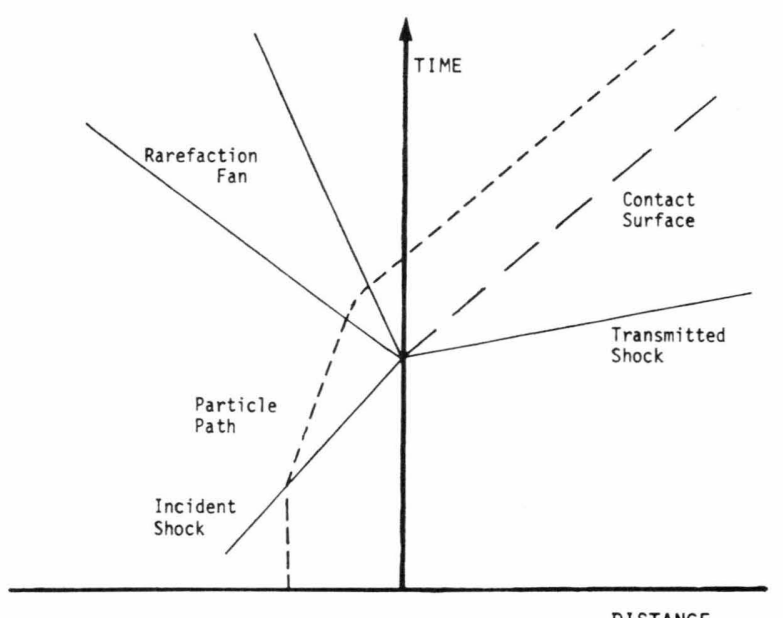

Fig. $2 \mathrm{~b}$

Fig. 2. a) $X-T$ diagram showing the predicted behaviour when a reflected shock is produced. b) $X-T$ diagram showing the predicted behaviour when a reflected rarefaction is produced.

can solve (1) and (2) relatively easily. Substituting (1) into (2) yields ${ }^{8}$

$\frac{1}{\varrho_{3}^{1 / 2}} \frac{(y z-1)}{(y z+\mu)^{1 / 2}}=\frac{1}{\varrho_{0}^{1 / 2}} \frac{y-1}{(y+\mu)^{1 / 2}}-\frac{1}{\varrho_{1}^{1 / 2}} \frac{z-1}{(z+\mu)^{1 / 2}} y^{1 / 2}$

where $\mu=(\gamma-1) /(\gamma+1)$.

If a reflected shock wave exists $(z>1$ to $z \approx 1)$, then

$$
\frac{1}{Q_{1}^{1 / 2}} \frac{(z-1)}{(z+\mu)^{1 / 2}} y^{1 / 2}>0 .
$$

Equations (3) and (4) imply that for the existence of a reflected shock wave $\varrho_{3}>\varrho_{0}$.

Similarly ${ }^{8}$, for a reflected rarefaction wave (2) becomes, putting

$$
\begin{aligned}
& A=2(1+\mu)^{1 / 2} /[(\gamma-1)(1-\mu)]>0 \\
& A y^{1 / 2}\left(1-z^{(\gamma-1) / 2 \gamma}\right)=\left[\begin{array}{l}
\varrho_{1} \\
\varrho_{3}
\end{array}\right]^{1 / 2} \frac{(y z-1)}{(y z+\mu)^{1 / 2}} \\
&-\left[\begin{array}{l}
\varrho_{1} \\
\varrho_{0}
\end{array}\right]^{1 / 2} \frac{(y-1)}{(y+\mu)^{1 / 2}} .
\end{aligned}
$$

If a reflected rarefaction wave exists $(z<1$ to $z \approx 1$ ), then

$$
\varrho_{0} \frac{(y z-1)}{\varrho_{3}} \frac{(y z+\mu)^{1 / 2}}{(y-1)} \frac{(y+\mu)^{1 / 2}}{\left(O_{1}\right.}
$$

(6) can be true only if $\varrho_{3}<\varrho_{0}$. Thus the above simplified derivation shows that for perfect gas conditions, a reflected shock must form if the incident shock wave moves from a rarer medium to a denser gas. A reflected rarefaction wave develops when the incident shocks move from a denser to a rarer gas.

Ford and Glass ${ }^{4}$, have derived analogous results for a boundary between two dissimilar ideal gases at different pressures by assigning each a different $\gamma$. Their results showed that the transition from reflected shock to reflected rarefaction depended not only upon the densities and natures of the gases involved, but also (weakly) on the initial shock velocity. For density discontinuities as large as those at the plasma boundary and shock Mach Nos. above 1.3, the above ideal gas analysis is adequate for qualitative predictions.

A brief summary by Emmens ${ }^{3}$, uses a technique similar to that of Ford and Glass, and is intended for ideal gases. Bowman and Whittaker ${ }^{6}$, studied colliding shocks in arcs using a single effective $\gamma$, but this was only valid because they used weak shocks, and their ionization and dissociation times were so long that ionization and dissociation could be considered to be frozen. For quantitative results in real gases, especially plasmas, none of these methods is adequate.

In our case 3 values of $\gamma$ would be required for each calculation, one for each of the regions, and one for the gas behind the reflected shock (or rarefaction). This last $\gamma$ is a function of the initial conditions, and the initial shock strength. Further, each $\gamma$ depends not only on the initial, but also on the final state of the gas, so extraction of an effective $\gamma$ from a table of thermodynamic properties cannot give accurate results, except for very weak shocks.

Ahlborn and Salvat ${ }^{9}$, developed what they called a quasi-analytic solution to the Rankine Huginiot 
equations using 2 gamma-like quantities to describe the state of the gas in front of and behind a single shock wave. Their method is graphical and iterative unless both the initial conditions and the final pressure are known, and it is valid theoretically only for shock Mach numbers greater than about 12 (strong shocks). The method is based on tabulated values of density and the gamma-like quantity $g$ as a function of temperature and pressure.

A comparison has been made between our results and those of Ahlborn and Salvat for Mach numbers up to 24 . For ideal gases comparison was also made with the algebraic expressions giving temperature and density. Strachan and Ahlborn ${ }^{5}$ used the method described above as part of the analysis of a shock front containing an energy source. They collided the first shock with another which contained no energy source (called the Swing probe). The calculation required knowledge of the conditions in the unshocked gas, initial and final velocities of both shocks and the velocity of the resultant contact surface which separates the regions behind the two resultant shocks. It depended on the probing shock being strong.

Thus all methods employed previously have restricted validity. We chose an iterative solution of the conservation equations, using a digital computer. The present paper describes shock refraction, but shock reflection and collision have also been studied. Our general method is more powerful than that of Strachan and Ahlborn in that knowledge of the initial conditions plus three of the five velocities they need would be sufficient to find all unknown quantities connected with the collision.

\section{Calculations for Reflected Shock Case}

The basic shock jump conditions, derived from the conservation of mass, momentum and energy are used in the following form.

$$
\begin{aligned}
P_{1}-P_{0} & =\varrho_{0} U u, \\
\varrho_{1} & =\varrho_{0} /(1-u / U), \\
H_{1}-H_{0} & =u U-\frac{1}{2} u^{2} .
\end{aligned}
$$

The subscripts 0 and 1 refer to conditions in front of and behind the shock respectively and $H$ is specific enthalpy.

It is also necessary to know the thermodynamic properties of the gas. The two relations

$$
H=H(T, P),
$$

$$
\varrho=\varrho(T, P)
$$

are used, and are obtained in numerical form from tables by Cambel, Drellishak et al. ${ }^{10,11}$.

All initial thermodynamic conditions, and the incident shock velocity $U_{1}$ are known. The calculation involves several coupled iterations and proceeds in two stages.

In the first stage the conditions behind the incident shock are iteratively calculated as follows:

(i) A convenient value is chosen for the gas velocity $u_{0}$ behind the incident shock $\left(U_{1}\right)$.

(ii) $u_{1}$ and $U_{1}$ are substituted into Eqs. (7) and (8) and values for $\varrho_{1}$ and $P_{1}$ found. Equations (10) and (11) are used to find a value for the enthalpy behind the initial shock, say $H_{1}{ }^{*}$.

(iii) The enthalpy behind the initial shock is also calculated from Equation (9). Call this $H_{1} \cdot H_{1}$ and $H_{1}{ }^{*}$ are compared.

(iv) If $H_{1}$ and $H_{1}{ }^{*}$ are not closely equal $u_{1}$ is adjusted to make them more nearly equal, and steps (ii) and (iii) repeated until $H_{1}=H_{1}{ }^{*}$.

The second stage in the calculation is a double nested iterative loop leading to a specification of conditions in regions 2 and 4 (see Figure 2). The steps may be summarised as follows.

(v) Based on refraction of a strong shock in an ideal gas an estimate is derived for the pressure behind the transmitted and reflected shocks $\left(P_{2}=\right.$ $\left.P_{4}\right)$.

(vi) The transmitted gas velocity $u_{4}$ is assumed to be a convenient fraction $(k)$ of the transmitted shock velocity $U_{4}$ and then a value for $U_{4}$ is found from

$$
u_{4}=k U_{4} \quad \text { and } \quad u_{4} U_{4}=\left(P_{4}-P_{3}\right) / \varrho_{3} .
$$

The latter of these equations is Eq. (7) re-arranged and referred to the regions under consideration. A value for $\varrho_{4}$ (the density behind the transmitted shock) is found from Eq. (8) referred to regions 3 and 4 .

(vii) Equations (10) and (11) are used to find a value for the enthalpy in region $4\left(H_{4}{ }^{*}\right)$ while Eq. (9) (referred to regions 3 and 4) is used to find another value $H_{4}$.

(viii) The value of $k=\left(u_{4} / U_{4}\right)$ is varied and steps (vi) and (vii) repeated until $H_{4}=H_{4}{ }^{*}$.

(ix) The velocity of the gas behind the transmitted shock (which is equal to that behind the reflected shock in laboratory co-ordinates) is trans- 
formed into co-ordinates fixed in the gas behind the initial shock.

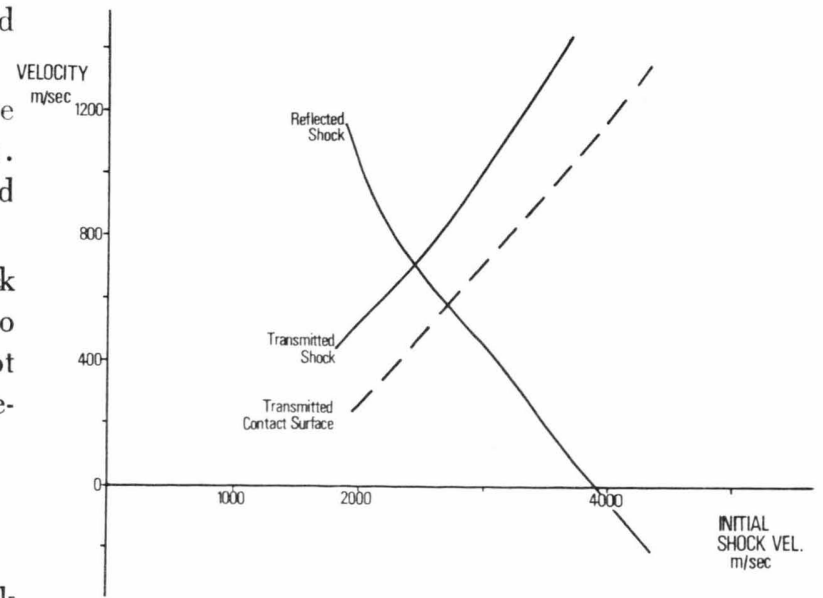

Fig. 3. Reflected and Transmitted Shock Velocities with Transmitted Contact Surface Velocity as a function of initial shock velocity. Initial conditions are 30 Torr with plasma temperature of $8250^{\circ} \mathrm{K}$ and cold gas temperature of $300^{\circ} \mathrm{K}$. results compared with those of Ahlborn and Salvat. For ideal gases a comparison was also made with equations from Wright ${ }^{12}$ :

The density as determined from

$$
\varrho_{1}=\varrho_{0} M^{2} /\left(1-u+u M^{2}\right)
$$

was compared with our results and those of Ahlborn and Salvat. Our results agreed with the equation, but Ahlborn and Salvat had too high a value at low Mach numbers, coming to within $5 \%$ of the others above Mach 10. Temperatures as calculated by Ahlborn and Salvat were systematically 7\% higher than those calculated by our method and from the equation:

$$
T_{1}=\left(T_{0} / M^{2}\right)\left[u\left(M^{2}-1\right)+M^{2}\right]\left[u\left(M^{2}-1\right)+1\right] .
$$

For real gases Ahlborn and Salvat's density figures vary from $10 \%$ low to $15 \%$ high by comparison with our method. Reasonable agreement seems to occur after Mach 20. The results for temperature differ by up to $5 \%$. Analysis of the equations used by Ahlborn and Salvat indicates that their results ought to agree with our more exact analysis for Mach numbers greater than 12. There is not sufficient information in their paper to allow us to see why this is not so but it may be that $g$ is not well enough known as a function of pressure (they show curves of $g$ vs. $T$ for pressures differing by factors of 10). The systematic error for ideal gases could result from a wrong value of sound speed. The value quoted in the paper is incorrect.

Typical results of the refraction calculations are shown in Figs. 3, 4 and 5. The sign convention used

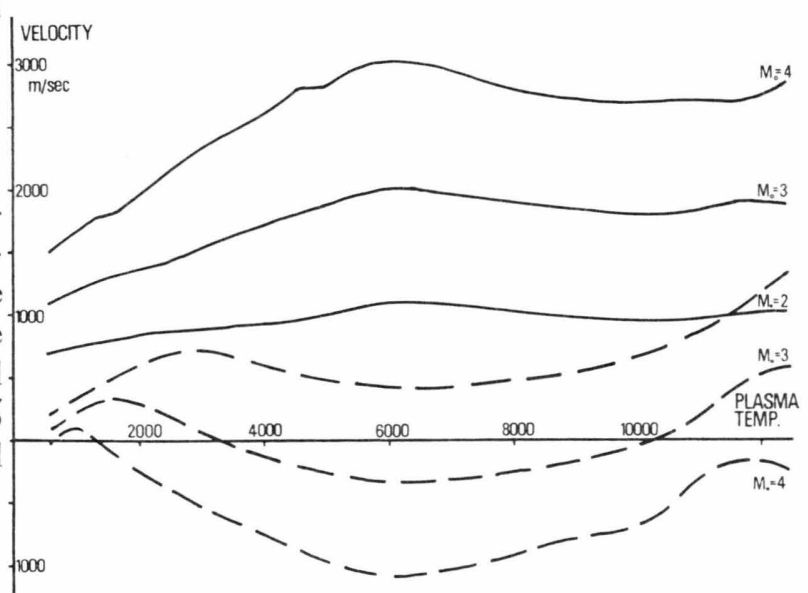

Fig. 4. Variation of transmitted and reflected shock velocities as a function of plasma temperature at 30 Torr. The cold gas temperature is $300^{\circ} \mathrm{K}$ and $\mathrm{Mo}$ is the initial shock mach no. — transmitted shocks; - - reflected shocks.

for velocity is that the direction in which the name of a wave suggests the wave should go is positive. So for a transmitted wave the positive direction is the same as for the incident wave, while for a reflected wave positive direction is opposite to that of the incident wave.

The most striking general conclusion is that the "reflected wave" need not always be reflected. It may under some conditions go forward, in which case the phenomenon would be better described as the splitting of the incident wave into two forwardgoing waves. Unfortunately mixing of driver and 


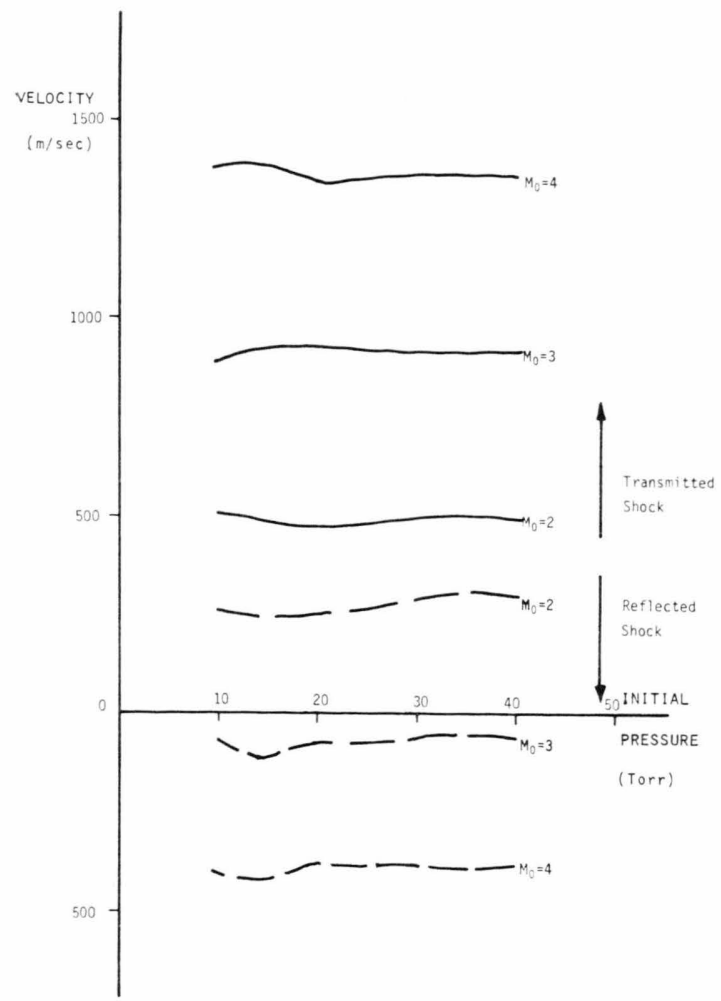

Fig. 5. Variation of transmitted and reflected shock velocities as a function of initial pressure. The plasma and cold gas temperatures are $8250^{\circ}$ and $300^{\circ}$ respectively.

test gases in our experimental apparatus prevents us from verifying this experimentally.

Figure 3 indicates the reflected and transmitted shock velocities together with transmitted contact surface (i. e. gas) velocity, as a function of initial shock velocity. The initial pressure for this case was 30 torr with the plasma at $8250 \mathrm{~K}$ and cold gas at $300 \mathrm{~K}$. It is clear that the non-linearity of the results is most obvious for the reflected wave.

Figure 4 shows the variation of transmitted and reflected shock velocities as a function of initial plasma temperature. The cold gas is held at $300^{\circ} \mathrm{K}$ and the initial pressure at 30 torr. Results are presented for three different initial shock mach nos. $\left(M_{0}\right)$ of 2, 3 and 4 . Comparison with Fig. 3 shows that for a fairly wide range of initial temperatures the resulting velocities depend much more on initial shock velocity than on plasma temperature. This applies particularly to the transmitted shock.

Figure 5 shows the behaviour of the reflected and transmitted shock velocities as the initial pressure is varied. Once again results are shown for initial shock mach. nos. of 2, 3 and 4 and the plasma and cold gas temperatures are $8250{ }^{\circ} \mathrm{K}$ and $300^{\circ} \mathrm{K}$ respectively. It can be seen that the transmitted and reflected shock velocities are relatively insensitive to changes in initial pressure.

\section{Analysis and Discussion of Results}

\section{(a) Plasma to Cold Gas Refraction}

Figure 6 a shows a typical streak record for the above case and Fig. $6 \mathrm{~b}$ shows its interpretation using the assumption that bright fronts on streak photos may represent either shock waves or contact surfaces.

Cloupeau ${ }^{13}$ has shown that when an electrothermal shock is launched into a cold gas the shock front is non-luminous while the contact surface shows up as a sharp bright line. Campbell et al. ${ }^{14}$ showed analogous results in a different situation. It seemed likely that the bright front in the cold gas in this case was also the contact surface.

This likely interpretation was checked using a reflector plate which could be placed anywhere in the cold gas or some way into the plasma. If a bright front is reflected on the reflector it cannot be preceded by a non-luminous shock. If the front is reflected some distance from the reflector then there must be a non-luminous wave ahead of it. The position of the reflector is indicated in Figure 6. This shows quite clearly the presence of a non-luminous shock in the cold region. The only reasonable explanation for the bright front is that it is a contact surface (see Figure 2). As the reflector plane is moved closer to the plasma the distance between the bright vertex A (in Fig. $6^{*}$ ) decreases and becomes undiscernable when the reflector is about $6 \mathrm{~cm}$. from the plasma. Reflection of the bright front occurs on the reflector when the reflector is in the plasma. Thus the bright line in the plasma region must be the incident shock front and the backward going front is the reflected shock.

For very low incident shock velocities two for. ward going bright fronts are recorded, see Figure 7 . As the incident shock velocities increase the two fronts coalesce into one. This suggested that the first bright front in the low velocity cases was the shock front, and the second was a contact surface. The disappearance of the contact surface is due to mixing of the driver and test gases.

* Fig. 6, 7, 9 see p. 1674 a-c. 
Theoretical and experimental results are compared in Figure 8. It will be seen that while refracted shock contact surface velocities agree well with theoretical calculations, reflected shock speeds do not, and these turn out to be higher than calculations predict. This is because the gas behind the initial shock has been mixed with the expanded driver and has become less dense. Unfortunately in the cases where both the incident shock and contact surface are present, the reflected shock is not strong enough to be visible. However, as shock velocities tend towards the conditions where separation occurs (i. e., low incident shock velocities), so the reflected shock velocity tends towards the expected value.

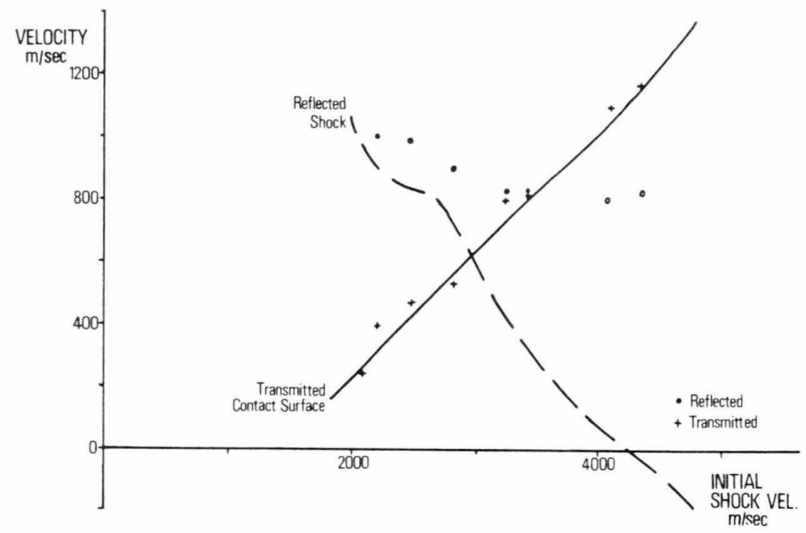

Fig. 8. Comparison of theoretical and experimental results for hot to cold refraction. Initial conditions are pressure 10 Torr, plasma temperature $9400{ }^{\circ} \mathrm{K}$ and cold gas temperature $300^{\circ} \mathrm{K}$; experimental points shown as $+\cdot \cdot$.

\section{(b) Formation Time of the Reflected Shock [for case (a)]}

Examination of the streak photos shows that the reflected wave does not appear as a sharp front immediately on formation. Using treatment given by Walsh ${ }^{15}$ an estimate of the time required to develop a shock wave, from a given initial acceleration in an atmosphere with spatial variations, has been calculated for the conditions of the gas at the transition region. The initial temperature in the transition region, is assumed to vary linearly between the preheated R.F. plasma and the cold gas (9000 to $300 \mathrm{~K}$ ). Hence the incident shock travels through a graded density region to become the refracted shock (or reflected shock), its speed also varying linearly between the two extreme limits over the distance of the transition region. This sets up a field of nonuniform velocity and pressure in the transition re- gion which in turn yields an accelerated gas flow sharpening into a shock wave.

Let the thickness of the transition region be $d$, and $\gamma$ be uniform throughout the region. The initial temperature at a point $x$ in the transition region would be $T_{x}=T_{0}-T_{d}(x / d)$ and the shock speed $U_{x}=U_{0}-U_{d}(x / d)$. Since the shock strength $y=$ $P / P_{0}=\left(U^{2} / R T\right)(1+\mu)$ where $P$ is the initial pressure behind the shock wave and $R$ is the gas constant, the pressure (at a point $x$ ) behind the shock would be,

$P_{x}=(1+\mu) P_{0}\left[\left(U_{0} d-U_{d} x\right)^{2} / \gamma R_{d}\left(T_{0} d-T_{d} x\right)\right]$.

The pressure difference $(\mathrm{d} P / \mathrm{d} x) \mathrm{d} x$ across the element of gas $\mathrm{d} x$, can be equated to the force per unit area on the element for obtaining the initial acceleration $a$ of the gas and is given by,

$$
\begin{aligned}
a=- & {[(1-u) / \gamma d]\{[(1-\mu) r R S} \\
& \left.+\mu W]\left(2 U_{d} S+T_{d} W\right) / S W\right\} .
\end{aligned}
$$

where $W=U_{0} d-U_{d} x$ and $S=T_{0} d-T_{d} x$. The negative sign in (11) indicates that the acceleration is in the opposite sense to $U$.

Assuming that the gas at the hot end of the transition region is moving back into a uniform atmosphere, the formation time $(t)$ of the reflected shock can be estimated from,

$$
t=2 c / a(1+\mu)
$$

where $c$ is sound velocity in the gas into which the reflected shock is propagating. [Examination of the streak photos suggests $2 \mathrm{~cm}$ as a reasonable estimate for d.] From (11) and (12), the approximated time of development of the reflected shock comes out to be $20 \mu \mathrm{sec}$ for the gas near the starting edge of the transition region and about $60 \mu \mathrm{sec}$ for the gas at the end of the transition region (cold end). The latter value is derived, again assuming that the gas molecules are being accelerated into a uniform atmosphere of temperature $T_{4}$, i. e., temperature behind the refracted shock. Examination of the streak photos shows that a reasonable estimate for the development time of the reflected shock (for the experimental condition of Fig. $6 \mathrm{a}$ ) is about $40 \mu \mathrm{sec}$.

It will be obvious that the mathematical model used here is crude, and that many refinements are possible. It was not considered worthwhile to do this, because formation time can only be measured subjectively from the streak photographs. 


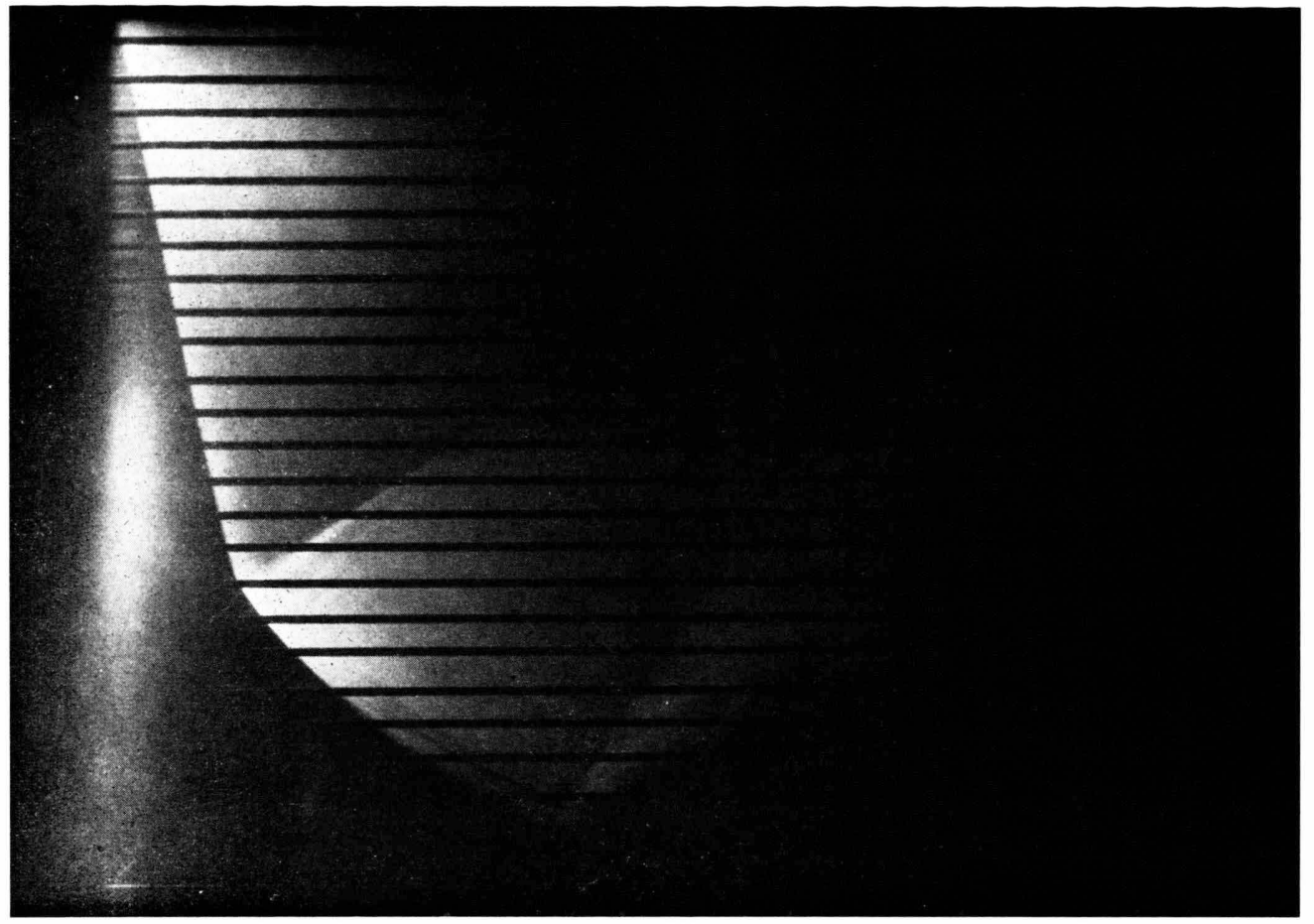

ơ
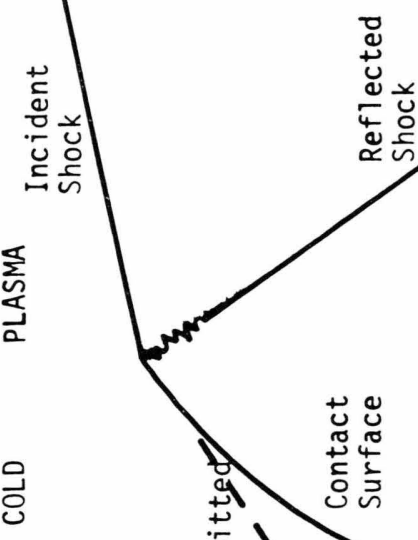

$\sum_{\frac{1}{n}}^{\frac{\pi}{2}}$

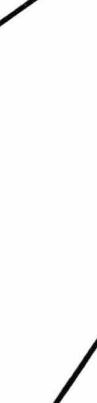

$\begin{array}{lll}1 & 1 \\ 8 & 8 \\ 8 & 0 \\ 0 & 0\end{array}$
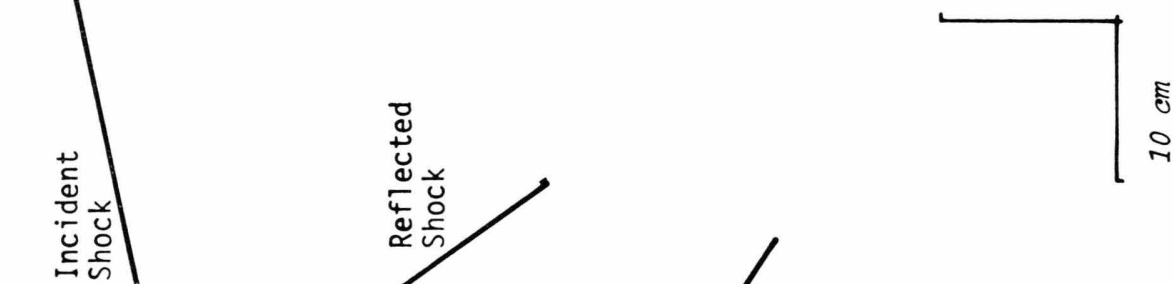

㝕美

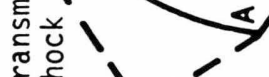

要总 


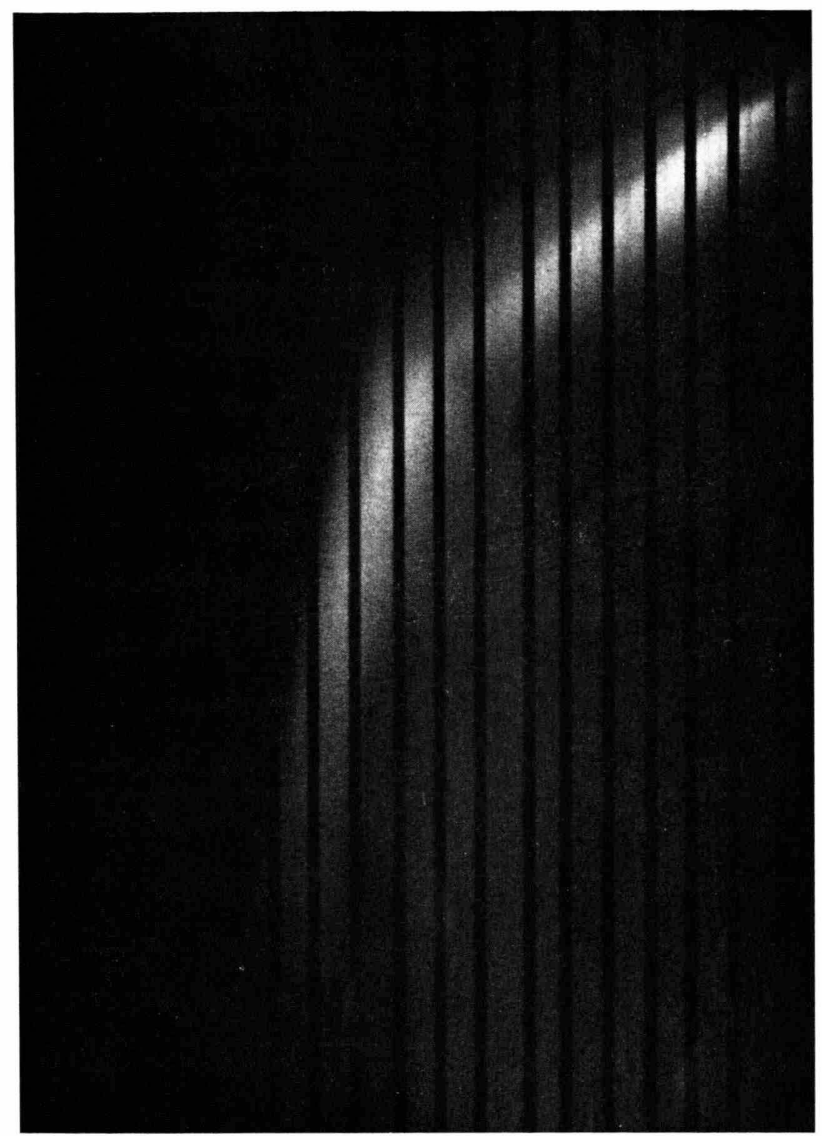

Fig. 7. Streak photograph of hot to cold refraction for low incident shock velocity $(2100 \mathrm{~m} / \mathrm{sec})$ where the contact surface remains distinct. 

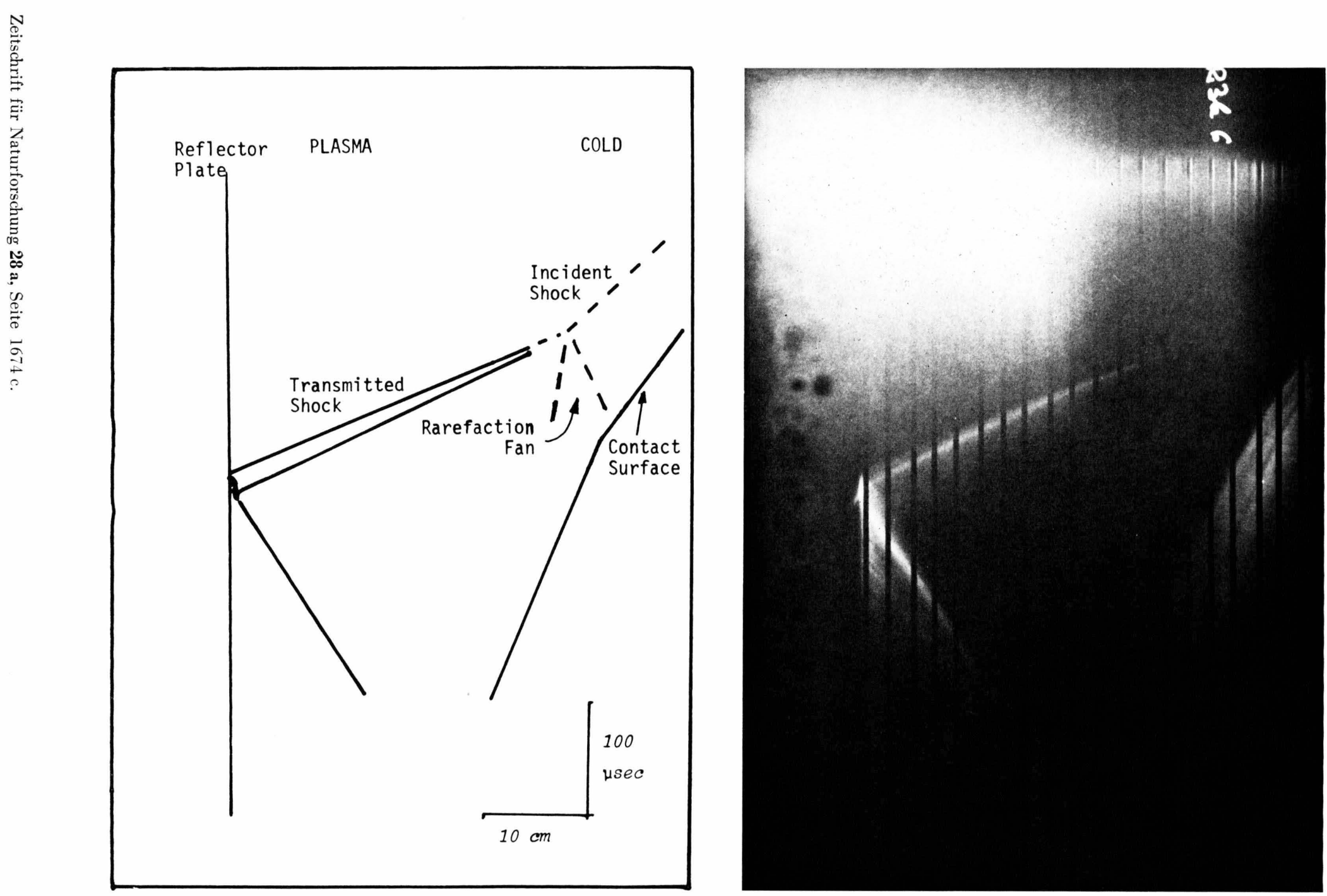

Fig. 9. Typical streak record of cold gas to plasma refraction together with its interpretation. 



\section{(c) Other Remarks on Plasma to Cold Refraction}

The behaviour of the observed striations in Fig. 6 a suggests they represent particle paths rather than waves. The velocity of the striations is very close to the velocity of the gas.

The image converter photographs confirm the conclusions drawn from streak photos. The only new feature that emerged from these pictures is the shape of the shock front. It is seen to be quite rounded. When the tube is used in the double ended mode (as in Ref. ${ }^{1}$ ) the shock front is straight. This can be explained if it is assumed that wall shear forces are the main cause of front curvature. The wall shear forces drop off sharply at a temperature a little above $10,000^{\circ} \mathrm{K}$, because of a sudden drop in the viscosity of argon. In the double ended mode gas temperatures are much higher than in the single ended mode because in the double ended mode even the R.F. plasma is arc heated.

\section{(d) Cold to Hot Refraction}

The bright incident front in the cold upstream gas has been shown by Campbell et al. ${ }^{14}$ to be a contact surface, preceded by a dark shock front. The luminous front that develops beyond the interface in the plasma region is assumed to be a shock front, since it is reflected at the surface of a reflector plane placed in the plasma.

A streak photograph and its interpretation is shown in Figure 9. A reflected rarefaction is expected at the cold gas-plasma interface but this cannot be seen on the photograph since it is diffuse and non-luminous.

Expected results have been calculated for this interaction, taking real gas effects into account. Experimental and theoretical results are compared in Table 1. It can be seen that there is excellent agreement between the two.

1 B. Campbell, H. K. Messerle, S. Ludvik, and A. D. Stokes, AIAA Journal 6, 1579 [1968] ; 10th Symposium on Engineering Aspects of Magnetohydrodynamics, M. I. T., March 26-28, Cambridge, Mass. 87-89, 1969.

2 D. W. George, L. P. Heffernan, and H. K. Messerle, Elect. Mech. Eng. Transactions of the Inst. of Engineers, Australia 6, 29 [1964].

${ }^{3}$ H. Polachek and R. J. Seeger, Shock Wave Interactions Fundamentals of Gas Dynamics (Ed. H. W. Emmens), pp. 482-525, Princeton University Press 1958.

4 C. A. Ford and I. I. Glass, J. Areo. Sci. 23, 189 [1956].

5 J. D. Strachan and B. Ahlborn, Canadian Physics 49, 1340 [1971].

${ }^{6}$ B. Bowman and D. Whittaker, British Applied Physics 17, 219 [1966].

7 V. H. Blackman and G. B. F. Niblett, Ionization Processes in Shock Waevs. Fundamental Data Obtained from Short
Table 1. Comparison of theoretical and experimental results for cold to hot refraction. Speeds are in metres/sec.

\begin{tabular}{lllll}
\hline $\begin{array}{l}\text { Initial } \\
\text { pressure }\end{array}$ & $\begin{array}{l}\text { Incident } \\
\text { shock vel. } \\
\text { calculated }\end{array}$ & $\begin{array}{l}\text { Incident } \\
\text { contact } \\
\text { surface vel. calculated }\end{array}$ & $\begin{array}{l}\text { Transmitted shock } \\
\text { velocity }\end{array}$ \\
\hline me Torr & 1510 & 1090 & 2800 & 2750 \\
20 Torr & 1575 & 1127 & 2880 & 2830 \\
& 1601 & 970 & 2756 & 2750 \\
30 Torr & 1340 & 950 & 2920 & 2880 \\
\hline
\end{tabular}

\section{Conclusion}

In the experiments described the passage of a shock wave from an R.F. plasma to a cold gas and from a cold gas to an R.F. plasma has been observed.

A computer-aided theoretical analysis of onedimensional shock refraction has been undertaken. The method has wider application than those published previously. In the regions relevant to our experiments the numerical analysis indicates that initial density ratio, and initial pressure play a much smaller role in determining final shock velocities than does the initial shock velocity.

The major part of the experimental work was carried out using a rotating mirror streak camera, yielding $x$ - $t$ diagrams of shock propagation. The various fronts on these $x$ - $t$ diagrams have been identified using a refraction model. The refraction calculations described above predict quantitatively the result of the passage of a shock wave through the plasma-cold gas interface.

\section{Acknowledgements}

The authors would like to express their gratitude to Mr. B. Campbell and Dr. M. Sakuntala for their helpful advice. The work is supported by a grant from the Australian Research Grants Committee.

Tube Expts. (Ed. A. Ferri), pp. 221-241, Pergamon Press 1961.

8 S. Paterson, Proc. Phys. Soc. London 61, 119 [1948].

${ }^{9}$ B. Ahlborn and M. Salvat, Z. Naturforsch. 22 a, 260 [1967].

10 K. S. Drellishak, C. F. Knopp, and A. B. Cambel, Partition Functions and Thermodynamic Properties of Argon Plasma, AEDC-TDR-63-146, Aug. 1963.

11 K. S. Drellishak, D. P. Aeschliman, and A. B. Cambel, Tables of Thermodynamic Properties of Argon, Nitrogen and Oxygen Plasma, AEDC-TDR-64-12, Jan. 1964.

12 J. K. Wright, Shock Tubes, Methuen 1961.

13 M. Cloupeau, Phys. Fluids 6, 679-88 [1963].

14 B. Campbell, D. W. George, and H. K. Messerle, Aust. J. of Phys. 22, 23-41 [1969].

15 E. K. Walsh, Phys. Fluids. 12, 757-63 [1969]. 